\title{
Observers' sensitivity to dynamic anomalies in collisions
}

\author{
MARY KISTER KAISER \\ NASA Ames Research Center, Moffett Field, California \\ and \\ DENNIS R. PROFFITT \\ University of Virginia, Charlottesville, Virginia
}

\begin{abstract}
The present study examined observers' ability to discriminate canonical and dynamically anomalous collisions that were presented in either frictionless or frictional systems. Whereas previous research has provided qualitative demonstrations that dynamic information can be extracted from visual events, the current study provides a parametric assessment of observers' sensitivity to dynamic invariants. Our findings indicate that observers are competent when viewing both familiar, terrestrial (frictional) systems and unfamiliar but computationally simpler, 0-G (frictionless) systems. Thus, our sensitivity to these dynamic invariants in visual events is robust in natural systems whose dynamic properties differ from those of the environment in which we evolved and developed.
\end{abstract}

The present study examined the perception of kinetic information in simple collisions. The term kinetic refers to the causes of object motions, and thus includes considerations of forces acting upon objects that have mass. When an event such as a collision is viewed, proximal stimulation consists not of forces and masses, but rather of patterns of motion, or kinematic information. In perceiving kinetic information, the perceptual system must rely on relevant regularities in proximal motion patterns.

Within the framework of Newtonian mechanics, the recovery of kinetic relationships from kinematic information can be achieved through the implementation of conservation laws. For collisions, the relevant laws are those of momentum conservation.

Consider the collision depicted in Figure 1. In the left panel, ball $A$ is shown moving toward ball $B$ along a vector path $v_{i a}$. In the middle panel, the instantaneous moment of impact is depicted, and in the right panel, the two balls are shown moving along their postcollision trajectories. The laws of momentum conservation state that the momentum of a system cannot be changed without the application of an external force. Thus, without external influences, the precollision momentum (mass $X$ velocity) of ball $A$ must be equal to the sum of $A$ and $B$ 's postcollision momenta. The initial kinematic information in this event consists of the velocity vector $\left(v_{i a}\right)$, but, without

\footnotetext{
This research was supported by NASA University Consortium Grant NCA2-87. Portions of this paper were presented at the Psychonomic Society 26th Annual Meeting, Boston, November 1985. We would like to thank Larry Beck of Sterling Software for his excellent programming support and Jim Johnston and Beth Wenzel for their helpful comments on an earlier draft of this paper. Requests for reprints should be sent to Mary Kaiser, NASA Ames Research Center, Mail Stop 239-3, Moffett Field, CA 94035.
}

knowledge of the masses involved, the postcollision motions cannot be predicted. If the pre- and postcollision velocities are known, however, the relative masses of the balls are uniquely specified by momentum conservation (Runeson, 1977). For the event shown in Figure 1, $\left(m_{a} * v_{i a}\right)=$ initial momentum of the system, and $\left(m_{a} * v_{f a}\right) * \cos (\alpha)+\left(m_{b} * v_{f b}\right) * \cos (\beta)=$ postcollision momentum, $\left(m_{a}+m_{b}\right) * v_{a b}$ where $v_{a b}$ is the postcollision resultant velocity vector. Momentum conservation requires that:

$$
\left(m_{a} * v_{i a}\right)=\left(m_{a} * v_{f a}\right) \cos (\alpha)+\left(m_{b} * v_{f b}\right) \cos (\beta),
$$

which can be rewritten:

$$
m_{a} / m_{b}=v_{f b} * \cos (\beta) /\left[v_{i a}-v_{f a} * \cos (\alpha)\right] \text {. }
$$

Thus, through the application of conservation laws, kinetic relationships (in this case, relative masses) can be recovered from purely kinematic information (velocity vectors). If one knows the pre- and postcollision velocities in any collision, the relative masses of the objects involved can be determined. These equations also show that the direction of linear momentum remains unchanged by the collision.

Untrained adult observers are able to make reliable relative mass judgments after viewing collisions (Kaiser \& Proffitt, 1984; Todd \& Warren, 1982), as can children by 7 years of age (Kaiser \& Proffitt, 1984). Does this imply that the perceptual system implements processing rules embodying the laws of momentum conservation? It is possible that the perceptual system has internalized such natural contraints (Shepard, 1984). It seems equally plausible, however, that perceptual processing relies upon more context-specific heuristics. Todd and Warren suggested that the perceptual system relies on a limited set 


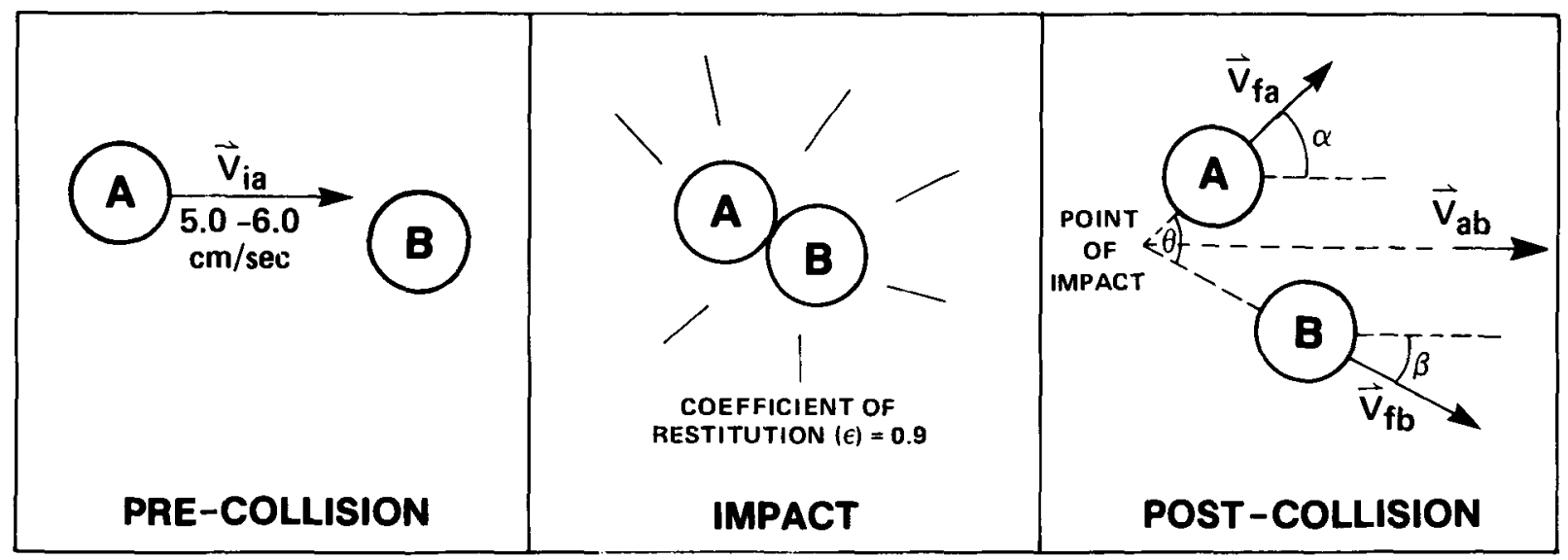

Figure 1. Diagram of collision used as experimental stimulus. Alpha is the postcollision vector angle of the striking ball, $\beta$ is the angle of the struck ball, and $\theta$ is the sum of $\alpha$ and $\beta$.

of the available information, and that it may employ heuristics specific to the terrestrial environment in which we live.

The present experiment was designed to provide more precise assessments of observer accuracy in recovering kinetic information from collisions; such assessments are required before much headway can be made in specifying the nature of the processing constraints employed. At present, the experimental evidence consists of two rather qualitative demonstrations of observer competence. Todd and Warren (1982) asked observers to make relative mass judgments for head-on collisions and found above-chance performance for each of the four mass ratios employed. (The smallest ratio used was 1.25.) Kaiser and Proffitt (1984) used only one mass ratio (approximately 2.8 ), and also found above-chance performance for observers ranging in age from 7-year-olds to adults. (The 5-year-olds they tested did not perform significantly better than chance.) Kaiser and Proffitt also asked their observers to judge whether each collision was a possible or impossible one, that is, whether or not it violated natural laws. Some of their collisions were natural sequences and others were contrived so as to violate the laws of momentum conservation. All of their observers, from children of 5 years of age to adults, showed above-chance performance on this task.

Although these demonstrations provide evidence that the recovery of kinetic information from collisions is within the competencies of the human perceptual system, neither of these studies provides much information about the accuracy with which these kinetic judgments can be made. Moreover, we do not know whether judgments are, in general, veridical or biased. As has been found for many other aspects of perceptual processing, kinetic information processing may reflect inherent perceptual biases, in this case biases not predicted by the conservation laws of physics.
The present experiment was designed to assess observer sensitivities to kinetic relationships in collisions, such as that depicted in Figure 1, in which a stationary ball is struck by a moving one. Various kinematic parameters were manipulated and, using a staircase procedure, observers were required to judge whether each event appeared to be natural or unnatural. In this manner, we determined a range for kinematic variables in which events appeared to be natural. The following variables were manipulated:

The presence or absence of external, terrestrial forces. The conservation equations given above ignore external frictional forces encountered on earth. If the balls roll on a surface within a gravitational field, then the equations become a good bit more complicated by frictional forces that produce continuous decelerations. Naturalness judgments were made for events that simulated both the presence and absence of friction. The latter events appeared as collisions occurring in a zero-gravity (0-G) environment, such as that of outer space. It was felt that the absence of friction might make the events appear so unfamiliar as to disrupt naturalness judgments. This possibility follows from Todd and Warren's (1982) hypothesis that kinetic sensitivities may be guided by context-specific heuristics that evolved in the terrestrial environment. On the other hand, it is possible that the greatly simplified kinematics of the frictionless collisions will facilitate the recovery of kinetic relations. Such a result would indicate that, at least for the collision type examined here, appreciation of the relevant natural laws is not specific to terrestrial contexts.

Conservation of momentum direction. As previously stated, the direction of the postcollision resultant vector must be the same as the initial velocity vector of the striking ball. To assess sensitivity to this rule, we varied the direction of the postcollision vector. Moreover, our stimulus manipulation allowed us to determine whether judg- 
ments were based on the direction of the resultant vector or on the postcollision trajectory of either of the two balls involved. Furthermore, we assessed the impact of distorting postcollision velocity vectors relative to the angle of impact while maintaining a canonical orientation of the resultant vector.

Conservation of momentum magnitude. Velocity was proportionally added to or subtracted from the two balls' postcollision vectors to determine observers' sensitivity to conservation of momentum magnitude. We were particularly interested in whether observers would tolerate an instantaneous reduction in velocity at the point of impact as a surrogate for the gradual loss of velocity that occurs in frictional systems.

Temporal properties of impact. Temporal contiguity has long been viewed as an intrinsic property of causal events (Hume, 1748/1961). Michotte (1963) studied the effect of disrupting the temporal flow of events on causal judgments. His experiments, however, utilized a rather primitive display system that did not achieve a realistic dynamic simulation, and his studies were qualitative in nature. We sought to determine the influence of temporal disruption at the point of impact on naturalness judgments.

\section{METHOD}

\section{Observers}

Four men and 4 women participated in the study. Their ages ranged from 18 to 40 years (mean age $=31.9$ ). The observers were recruited among colleagues, research assistants, and the paid subject pool at the first author's institution.

\section{Display}

All stimulus events were displayed on a 33-cm diagonal RGB monitor driven by a MASSCOMP 550 minicomputer running in single-user, real-time mode. The stimulus events showed the collision of two balls represented by red, filled circles, $1 \mathrm{~cm}$ in diameter. The underlying collision algorithm assumed the balls to be of equal mass. (Since mass was not a variable under study, equal masses provided the simplest case.) When the event was initiated, one ball was always stationary in the center of the screen. The striking ball entered from the left side of the screen, traveled horizontally, and collided with the stationary ball at an oblique angle $\left(30^{\circ}\right.$ or $\left.40^{\circ}\right)$. A regular grid was present as background, with lines $1 \mathrm{~cm}$ apart. This grid background was used to minimize induced motion effects and to aid observers in trajectory angle estimation. An auditory cue signaled the beginning of each trial.

Six kinds of dynamic distortions were displayed: four of an angular nature, one temporal, and one involving momentum. The parameters of the angular distortions are illustrated in Figure 1. The first type of angular distortion involved a symmetric perturbation in the postcollision direction of both the striking ball $(\alpha)$ and the struck ball $(\beta)$. Thus, the sum of $\alpha$ and $\beta$ ( $\theta$ in Figure 1) remained constant, but was shifted about the natural, horizontal vectorsum orientation. The second type of angular distortion perturbed $\alpha$ while keeping $\beta$ at its natural postcollision magnitude. The third kind of distortion perturbed $\beta$ while keeping $\alpha$ canonical. The fourth kind of distortion expanded or contracted both $\alpha$ and $\beta$ proportionally to their natural magnitude. Thus, the vector-sum remained canonical (i.e., horizontal), but the magnitude of $\theta$ was perturbed. This fourth type of distortion was used to assess observers' sensitivity to contact angle; the resultant momentum vector is undistorted, but the vectors no longer correspond to the angle of incidence.
In the momentum distortions, velocity was added proportionally to the postcollision vector of each ball. That is, each postcollision vector was expanded or contracted by an equal proportion. Thus, the direction of the resultant vector was canonical, but its magnitude was increased or decreased. The temporal distortion disrupted the contiguity of the event; either the balls froze at the point of impact or the "struck" ball started its postcollision sequence prior to actual contact.

\section{Procedure}

The observers participated in two sessions, viewing events in a frictionless system in one and viewing objects with a frictional coefficient of 0.7 in the other. (This is about the frictional coefficient of steel on wood, like the shuffleboard games found in bars. We picked a coefficient representative of the range observed in everyday events.) Friction was implemented as a constant deceleration function of the objects' velocities. The order in which sessions were administered was counterbalanced across subjects. Each session consisted of three blocks, the first two containing angular distortions and the third consisting of momentum and temporal distortions. An interleaved staircase procedure was employed (Levitt, 1971). Each type of distortion generated four staircases, since the staircase could be either ascending or descending and could start at either the natural or grossly distorted point. Thus, eight interleaved staircases were run in each block. Each staircase was assigned one of two initial velocities $(5$ or $6 \mathrm{~cm} / \mathrm{sec})$ and one of two angle of incidence $\left(30^{\circ}\right.$ or $40^{\circ}$ ). Staircases were terminated after the eighth reversal.

From a viewing distance of approximately $50 \mathrm{~cm}$, the entire event subtended an angle of $23^{\circ}$. The observers were allowed free head and eye movement throughout the study. Each trial proceeded as follows: The grid and the stationary ball appeared on the screen, an auditory signal sounded, and the collision was presented, terminating when both balls exited the screen. An icon of the computer terminal's mouse then appeared on the screen, with instructions to press the left button if the event appeared to be natural or the right button if it looked distorted. The computer recorded the observer's response, calculated parameters for the next trial of that staircase, then proceeded to the next trial of the next staircase.

The observers were instructed that gravity did not influence the balls' trajectories. For the frictionless system, we suggested that observers view the events as if they were occurring in outer space (i.e., in the absence of gravity and friction). For the frictional system, we told the observers to view the events as if they were doing so looking down on a billiard table, with the trajectories neutral with respect to gravity.

\section{RESULTS}

Midrun estimates were calculated for the last four runs of each staircase; the average of these four values was used as the measurement of the acceptance threshold $\left(X_{50}\right){ }^{1}$ Each type of distortion has two thresholds associated with it: a positive threshold (i.e., the $+\Delta$ value at which the distortion is detected) and a negative threshold. Both the positive and negative thresholds have two estimates per subject: one from the ascending staircase, the other from the descending staircase.

Threshold estimates were averaged across staircase direction and observers (no significant effects were observed for either factor). Figure $2 \mathrm{a}$ illustrates the acceptance ranges for the four types of angular distortions. The distortions of $\alpha, \beta$, and $\theta$ can be either toward the implicit gravitational vector (clockwise, or negative) or against it (counterclockwise, or positive). The fourth type 
(a)

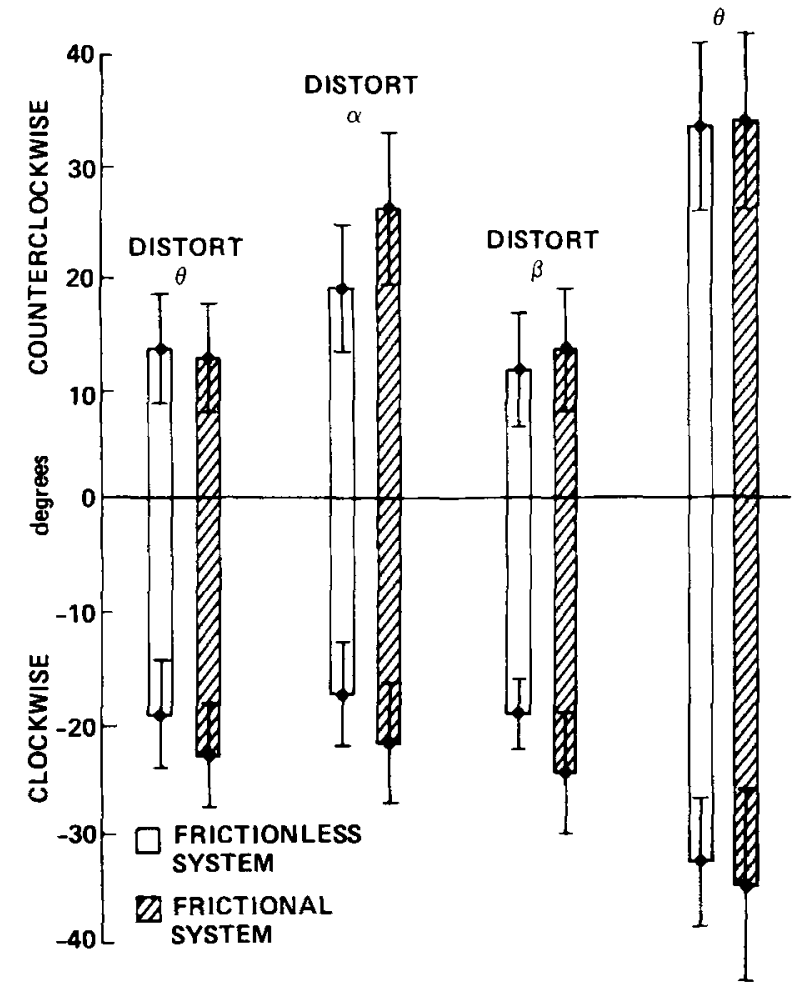

(b)

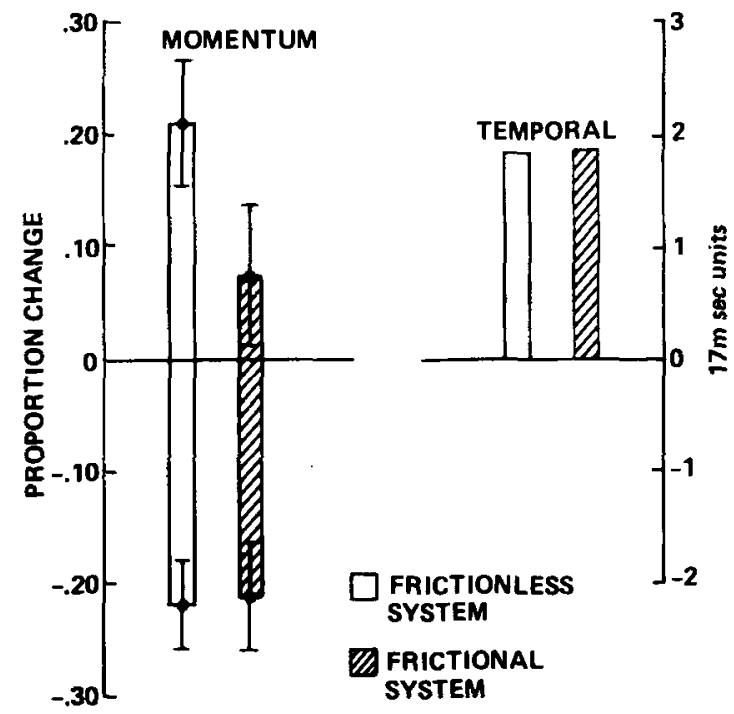

Figure 2. Acceptance ranges defined by averages of observers' thresholds for (a) angular distortions and (b) momentum and temporal distortions.

of distortion involves the expansion (positive degrees) or contraction (negative degrees) of $\theta$. Acceptance ranges for the momentum and temporal distortions are shown in Figure $2 \mathrm{~b}$. It should be noted that, due to the cycle rate of the collision animation program and display hardware $(60 \mathrm{~Hz})$, our temporal resolution is limited to $17 \mathrm{msec}$.
Thus, a more accurate description of the temporal distortion acceptance range is that delays longer than $2 \mathrm{cy}$ cles were not tolerated, and any premature initiation of the postcollision sequence was viewed as anomalous. Figure 3 shows the acceptance ranges for the first three kinds of angular distortions in terms of perturbation of the resultant momentum vector. Thus, the values for distortions of $\alpha$ alone and $\beta$ alone are adjusted to reflect the distortion of the entire system's momentum.

Acceptance ranges were compared for the systems with and those without friction. A significant difference in tolerance occurred only for the momentum distortions, with observers more accepting of momentum distortions in the frictionless system $[t(7)=1.68, p<.05]$.

Finally, the data were examined for any bias toward acceptance of angular distortions in the gravitational direction $\left(\left|-X_{50}\right|>\left|+X_{50}\right|\right)$. Although the observers were explicitly told that gravity would not affect the trajectories in either system, it is possible that the observers developed an implicit expectation of gravitational effects. Such a bias was found when $\theta$ was distorted $[t(7)=1.67$, $p<.05]$ and when $\beta$ was distorted $[t(7)=1.99$, $p<.05]$, but not when $\alpha$ was distorted.

\section{DISCUSSION}

Previous research has demonstrated a perceptual competence for the extraction of dynamic information from collisions. This competence is demonstrated in the absence of explicit knowledge of collision mechanics (Todd \& Warren, 1983). Indeed, even 7-year-olds exhibit sensitivity to invariants of collision dynamics. Furthermore, adults who have been exposed to the formalisms of mechanics nonetheless make more accurate judgments when viewing dynamic events than when reasoning from static representations (Kaiser \& Proffitt, 1984). The current study extends our understanding of dynamic event

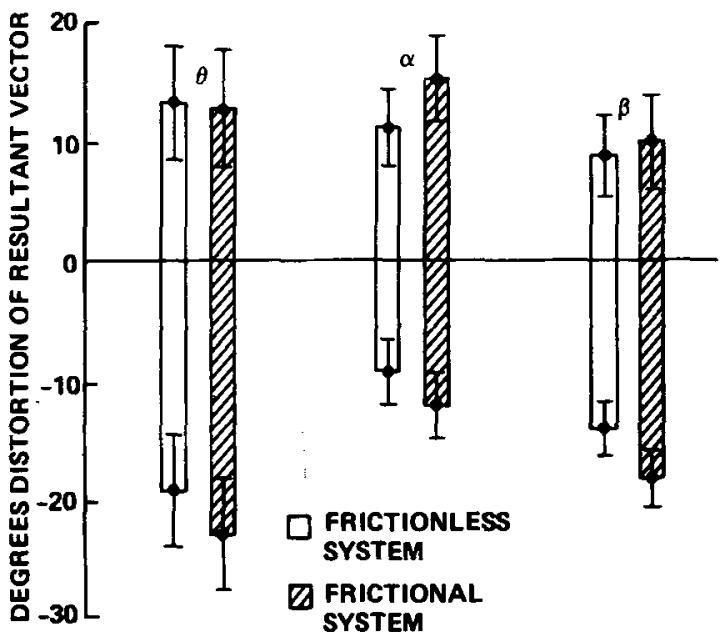

Figure 3. Acceptance ranges defined by averages of observers' thresholds for the distortions of the resultant momentum vector when $\theta, \alpha$, and $\beta$ are modified. 
perception by examining observers' sensitivities in a parametric manner and assessing whether observers' competencies are robust to nonterrestrial, yet dynamically canonical, environments. Our findings are considered in terms of the issues raised in the introduction:

The presence or absence of external, terrestrial forces. In general, observers performed with equal competence when viewing events in frictionless and frictional systems. The single exception to this was that observers were less tolerant of the addition of momentum to frictional systems. Otherwise, acceptance ranges were quite similar for the two systems.

As we suggested, one might have expected differential performance. The frictionless system is computationally less complex, since constant pre- and postcollision velocities are maintained. Hence, the perceptual system would be performing the necessary transformation on less complex information. Alternatively, one could argue that humans have evolved and developed in a terrestrial environment in which frictional forces are a major determinant of motion. Thus, observers could be expected to have a greater competence with frictional systems because of their experience with them. Our data suggest that neither simplicity nor familiarity wins the day. Observers demonstrate a fair degree of competence at extracting dynamic information from both systems. There is no evidence that nonterrestrial systems are viewed as being anomalous; observers may be more familiar with terrestrial events, yet they are equally competent at recognizing dynamic invariants in frictionless, $\mathbf{0 - G}$ systems. Observers do not equate terrestrial and natural; rather, judgments reflect an appreciation of general dynamic invariants.

Conservation of momentum direction. Observers' sensitivity to the distortion of the postcollision vectors did not differ significantly for the two balls (i.e., $\alpha$ vs. $\beta$ ). Thus, observers' decisions are not differentially based on either the striking or struck ball's trajectory. Moreover, observers are relatively insensitive to the correspondence of the angle of impact and the postcollision trajectories (i.e., expansions and contractions of $\theta$ ), at least for the displays we used.

Results were equivocal as to whether anomaly judgments were based on the distortion of the resultant vector or upon absolute angular distortion of the trajectories. The acceptance range for distortions of $\theta$ tended to be smaller than the acceptance ranges for $\alpha$ and $\beta$ in terms of absolute angular distortion, but larger than $\alpha$ 's and $\beta$ 's ranges in terms of distortion of the resultant vector. Neither comparison yielded significant differences.

Observers demonstrated more acceptance when the distortion was toward the gravity vector for two of the three angular distortions. Although observers were explicitly told that gravity was not an influence on the balls' trajectories, they may nonetheless have been more tolerant of distortions in the downward direction, or may have found such distortions more difficult to perceive. Such gravitationally anisotropic judgments need to be investigated more fully.
Conservation of momentum magnitude. Observers' acceptance ranges for momentum distortions are much larger than one would predict on the basis of thresholds for velocity change, which usually range from 5\%-10\% (McKee, 1981). Of course, judging momentum change is computationally more complex, since the summation of two velocity vectors is required. Also, ours is not a simple discrimination task, but rather requires a more complex naturalness judgment.

The instantaneous loss of momentum at the point of impact is viewed as equally anomalous in the frictionless and frictional systems; the temporally discrete loss of velocity is not an acceptable surrogate for the continuous loss function friction imposes.

Temporal properties of impact. Examination of the acceptance ranges from temporal distortions suggests that observers are more sensitive to disruptions in contiguity than Michotte's (1963) data would suggest. His studies found that observers would tolerate a delay of $100 \mathrm{msec}$ or more before the impression of "direct causality" was disrupted. Although the precision of our estimate is constrained by the update rate of our display, it is unquestionably much smaller than the earlier estimates. $\mathrm{Ob}$ servers are clearly very sensitive to disruptions in the temporal contiguity of simple physical events. Quite likely, Michotte's displays were not compelling representations of dynamic events and so did not fully capture observers' sensitivity to temporal parameters.

Taken as a whole, our data suggest that observers are able to distinguish canonical and anomalous events in both frictional and frictionless systems. Perceptual competencies are not constrained to environments of evolution and development. Some evidence suggests that observers' judgments may reflect a bias toward terrestrial conditions (i.e., the greater acceptance of distortions coincident with the gravitational vector), but their competence for recognizing natural, yet nonterrestrial, events is not compromised.

Thus, the human observer has a well-defined set of implicit criteria by which to judge whether or not a collision is a natural, momentum-conserving event. These criteria appear to be based on underlying dynamic invariants such that perceptual competence is robust to dynamic properties of the system and is not constrained by our terrestrial experience.

\section{REFERENCES}

HuME, D. (1961). An enquiry concerning human understanding. New York: Dolphin. (Original work published 1748)

Kaiser, M. K., Proffit, D. R. (1984). The development of sensitivity to causally-relevant dynamic information. Child Development, 55, 1614-1624.

LEVITT, H. (1971). Transformed up-down methods in psychoacoustics. Journal of the Acoustical Society of America, 49, 467-477.

MCKeE, S. P. (1981). A local mechanism for differential velocity detection. Vision Research, 21, 491-500.

MichotTE, A. (1963). The perception of causality. New York: Basic Books.

Runeson, S. (1977). On visual perception of dynamic events. Unpublished doctoral dissertation, University of Uppsala, Uppsala, Sweden. 
SHEPARD, R. N. (1984). Ecological constraints on internal representations: Resonant kinematics of perceiving, imagining, thinking, and dreaming. Psychological Review, 91, 417-447.

ToDD, J. T., \& WARREN, W. H. (1982). Visual perception of relative mass in dynamic events. Perception, 11, 325-335.

\section{NOTE}

1. The staircase methodology we employ allows us to determine the magnitude of the distortion parameter at which an observer judges the event to appear to be natural $50 \%$ of the time $\left(X_{50}\right)$. Thus, we employ a rather unconventional use of the construct signal: it is a dynamic anomaly sufficient to distinguish the event from the observers' implicit category of canonical events.

(Manuscript received September 19, 1986; revision accepted for publication March 20, 1987.) 